\title{
Noise Reduction in CdZnTe Coplanar-Grid Detectors
}

\author{
P. N. Luke, J. S. Lee, M. Amman, and K. M. Yu
}

\begin{abstract}
Noise measurements on CdZnTe detectors show that the main sources of detector-related noise are shot noise due to bulk leakage current and $1 / \mathrm{f}$ noise due to the detector surfaces. The magnitude of surface leakage current appears to have little or no effect on the detector noise. Measurements on guard-ring devices fabricated using gold-evaporated contacts show that the contacts behave as Schottky barriers, and the bulk current at typical operating voltages is likely dependent on the contact properties rather than directly on the material's bulk resistivity. This also suggests that the level of shot noise is affected by the detector contacts and not necessarily by the material's bulk resistivity. A significant reduction in the noise of coplanar-grid detectors has been obtained using a modified contact fabrication process.
\end{abstract}

\section{INTRODUCTION}

The energy resolution of semiconductor gamma-ray detectors depends to a large extent on the charge transport properties of the material as well as electronic noise associated with the detectors and amplifiers. For CdZnTe detectors, the effects of poor charge transport, which is especially severe for the holes, can be dealt with very effectively using the coplanar-grid technique. With this technique, it is possible to produce large-volume $\left(\sim 1 \mathrm{~cm}^{3}\right)$ $\mathrm{CdZnTe}$ gamma-ray detectors with good energy resolution $(\sim 2 \% \mathrm{FWHM}$ at $662 \mathrm{keV})$ and near full-volume efficiency $(\sim 90 \%)$ [1]. The energy resolution that can be achieved depends substantially on the uniformity of the CdZnTe material. The degree of non-uniformity in current CdZnTe materials varies widely, resulting in detectors with energy resolution ranging from $\sim 2 \%$ to $5 \%$ or more. Obviously, continuing effort is needed to understand and control material non-uniformity in order to improve the yield of highresolution detectors [2]. Besides material non-uniformity, electronic noise can also have a large effect on the overall energy resolution of these detectors. The typical electronic noise of $1-\mathrm{cm}^{3} \mathrm{CdZnTe}$ coplanar-grid detectors that we have fabricated has been consistently at the level of $\sim 10 \mathrm{keV}$ FWHM. This level of noise degrades the energy resolution of the detector at high gamma-ray energy, and becomes the dominant effect limiting resolution at low energy. The

Manuscript received November 16, 2001.

This work was supported by the U. S. Department of Energy, Office of Defense Nuclear Nonproliferation, Office of Nonproliferation Research and Engineering (NN-20), under Contract No. DE-AC03-76SF00098.

P. N. Luke, J. S. Lee, M. Amman and K. M. Yu are with the Lawrence Berkeley National Laboratory, Berkeley, CA 94720 USA. (first author email: pnluke@lbl.gov). relatively high level of noise compared to simple planar detectors can be attributed to the effects of the closely spaced electrode structures used on coplanar-grid detectors and the inter-grid voltage bias required during operation. The subtraction of signals from the two grid electrodes also increases the noise since the noise from the two separate amplification channels add in quadrature (assuming the noise from the two channels are not correlated).

In order to gain a better understanding on the origins of noise and to develop methods to reduce noise in coplanargrid detectors, we have previously studied the noise behavior of CdZnTe devices with guard-ring and strip electrode structures [3]. The results from these studies indicated that the dominant noise sources, besides the preamplifier (FET) noise, are shot noise from the detector's bulk leakage current and surface related $1 / \mathrm{f}$ noise. Contrary to conventional expectation, the surface leakage current of the detector does not appear to produce shot noise and therefore does not contribute significantly to the overall electronic noise, even though the surface current is usually much higher than the bulk current. These observations were made with detectors fabricated using fairly standard and commonly used processes. These results suggest that the bulk current rather than the surface current needs to be lowered in order to reduce the detector noise.

\section{BULK CURRENT}

If the contacts of a detector are truly ohmic, the bulk current would be determined by the bulk resistivity of the material, and the only way to decrease the shot noise would be to use materials with higher resistivity. However, the contacts being used on these detectors often do not behave as ideal ohmic contacts. Figure 1 shows the current-voltage (IV) characteristics of a CdZnTe detector with a guard-ring electrode structure. The overall dimension of the device is 10 $\mathrm{mm}$ X $10 \mathrm{~mm}$ X $10 \mathrm{~mm}$. The center contact has an area of 7.5 $\mathrm{mm} X 7.5 \mathrm{~mm}$, with a gap of $\sim 1 \mathrm{~mm}$ between it and the guard ring. The contacts were formed by Au evaporation onto the surfaces of the CdZnTe crystal, which have been etched in bromine-methanol solutions. The current from the guard ring shows a nearly linear relationship with the applied bias, which would lead one to believe that the contacts are ohmic. However, the current from the center contact is much lower in magnitude and it shows an initial rise and then flattens out at a certain voltage, a clearly non-ohmic behavior. These results indicate that the $\mathrm{I}-\mathrm{V}$ characteristic of the guard ring is 


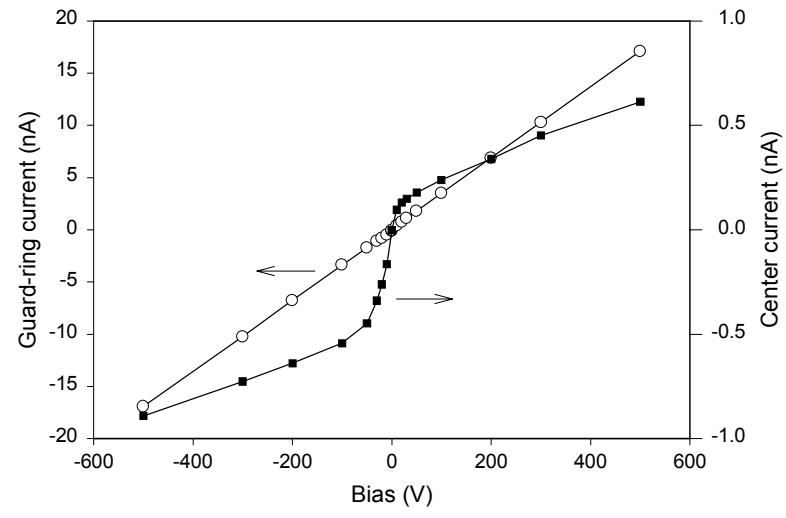

Fig. 1. I-V characteristics of a CdZnTe guard ring device. The device's dimension is $10 \mathrm{~mm}$ X $10 \mathrm{~mm}$ X $10 \mathrm{~mm}$. The center contact has an area of $7.5 \mathrm{~mm} \mathrm{X} 7.5 \mathrm{~mm}$ with a gap of $\sim 1 \mathrm{~mm}$ between it and the guard ring contact.

dominated by surface current and is not indicative of bulk conduction. This also means that the I-V characteristics measured on devices with full-area contacts should not be relied upon for determining bulk current or resistivity, since they are likely dominated by surface currents.

In Fig. 1, the measured current at the center contact consists of only bulk current, and the I-V characteristic is similar to that of a device with back-to-back Schottky barrier contacts. According to the simple thermionic emission model, the reverse current of a Schottky barrier device can be expressed as

$$
\mathrm{I}=\mathrm{I}_{\mathrm{s}}[\exp (-\mathrm{qV} / \mathrm{kT})-1],
$$

where $I_{s}$ is the saturation current, $q$ is the electronic charge, $V$ is the applied voltage (reverse bias), and $\mathrm{T}$ is the temperature. The magnitude of $I_{s}$ depends on the barrier height and temperature. From the expression, it can be seen that the current initially increases rapidly and approaches the saturation current $\left(I_{s}\right)$ at a relatively small voltage on the order of $\mathrm{kT} / \mathrm{q}$ or $0.026 \mathrm{~V}$ (at room temperature). At voltages above a few times $\mathrm{kT} / \mathrm{q}$, the current essentially reaches saturation and remains virtually constant at that level as bias increases.

Figure 2 shows the low voltage region of the I-V characteristic of another guard-ring device. Included in the figure is the current for an ideal Schottky barrier calculated using the above equation. In the calculation, $\mathrm{I}_{\mathrm{s}}$ was simply chosen to approximate the measured current so as to provide a qualitative comparison. Compared to the calculated current, the measured current shows a much slower initial rise and does not reach "saturation" until $\sim 2 \mathrm{~V}$. Also, the measured current continues to increase, although at a lower rate, as bias increases beyond "saturation". The difference in the initial rise of the current can be accounted for by including the bulk resistance of the CdZnTe material. In this region, the contact can supply a larger current, up to the level of the saturation current, but the bulk resistance (R) limits current flow to

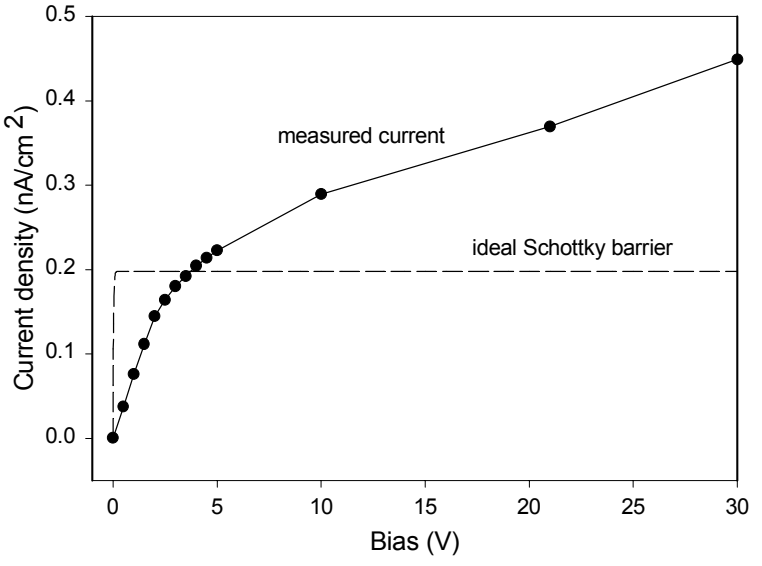

Fig. 2. I-V characteristics of the center contact of a CdZnTe guard-ring device compared to that of an ideal reverse biased Schottky barrier.

$\mathrm{I}=\mathrm{V} / \mathrm{R}$. The initial slope of the current voltage characteristics should therefore provide a true measure of the bulk resistivity of the material. After the current reaches "saturation", the contact can no longer supply the current needed to maintain the ohmic behavior. At that point, the current becomes limited by thermionic emission at the Schottky barrier. The rise in the measured current in the "saturation" region with increasing bias is commonly observed in practice in actual Schottky barrier devices. It may be caused by the lowering of barrier height due to the effects of image force or the existence of an interfacial layer, or it could be a result of carrier generation within the semiconductor [4].

If this model of the current-voltage behavior of the CdZnTe detector is correct, the bulk current under typical operating voltages for these devices depends to a large extent on the properties of the contacts. The bulk resistivity of the material may still have an effect on bulk current, but unless it is very high, the bulk current is largely limited by the contact. Therefore, it should be possible to change the bulk current by changing the properties of the contacts without necessarily using materials with higher bulk resistivity. For example, a contact with a larger barrier height should give a smaller bulk current, and that would lead to a lower level of shot noise.

\section{COPLANAR-GRID DETECTORS}

Since it is apparent that the contacts play an important role in determining bulk current and thereby shot noise, we investigated various contact fabrication processes in an attempt to lower the noise in coplanar-grid detectors. The conventional process that we use to fabricate these detectors involves mechanical lapping and polishing of the CdZnTe crystal followed by chemical etching in bromine-methanol solutions. Gold is then evaporated onto the etched surfaces of the crystal to form the electrodes. Shadow masks are used to define the coplanar-grid electrode pattern. In one of the modified processes that we have investigated, the detector electrodes are deposited onto a mechanically polished surface 
of the CdZnTe crystal without performing the brominemethanol etch. To measure noise, the detector is connected to the normal electronics used to perform gamma-ray spectroscopy. In this setup, each of the two grid electrodes is AC-coupled to a charge-sensitive amplifier, and the outputs of these two amplifiers are subtracted to give a net signal output. The net output is fed to shaping amplifiers with variable shaping times. Noise is measured at the output of the shaping amplifiers. During noise measurements, the detector is operated under typical bias conditions.

Figure 3 compares the measured noise as a function of peaking time from a coplanar-grid detector fabricated alternately using the two processes described above. From the noise and peaking time relationship, the different noise types can be extracted as shown in the figure. Series noise arises mainly from the preamplifier (FET) input noise, while the parallel noise and $1 / \mathrm{f}$ noise are due to bulk current shot noise and surface noise from the detector respectively [3]. It can be seen that with the modified fabrication process, both the parallel noise and the $1 / \mathrm{f}$ noise decreased. At the typically used peaking time of $4 \mu \mathrm{s}$, the total noise decreased from 970 e rms to 760 e rms. The current across the grid electrodes decreased substantially, from $\sim 200 \mathrm{nA}$ to $\sim 30 \mathrm{nA}$. The current is likely dominated by surface conduction, which, as we have found previously, does not produce significant noise. The reduction in parallel noise is likely due to a reduction in bulk current. This however cannot be confirmed since it is not possible to separately measure bulk and surface current between the grid electrodes in the coplanar-grid configuration. Additional measurements using guard-ring devices will be needed to clearly establish if the reduction in noise is indeed due to a reduction in bulk current.

Figures 4 and 5 show Cs- 137 and Co-57 spectra obtained

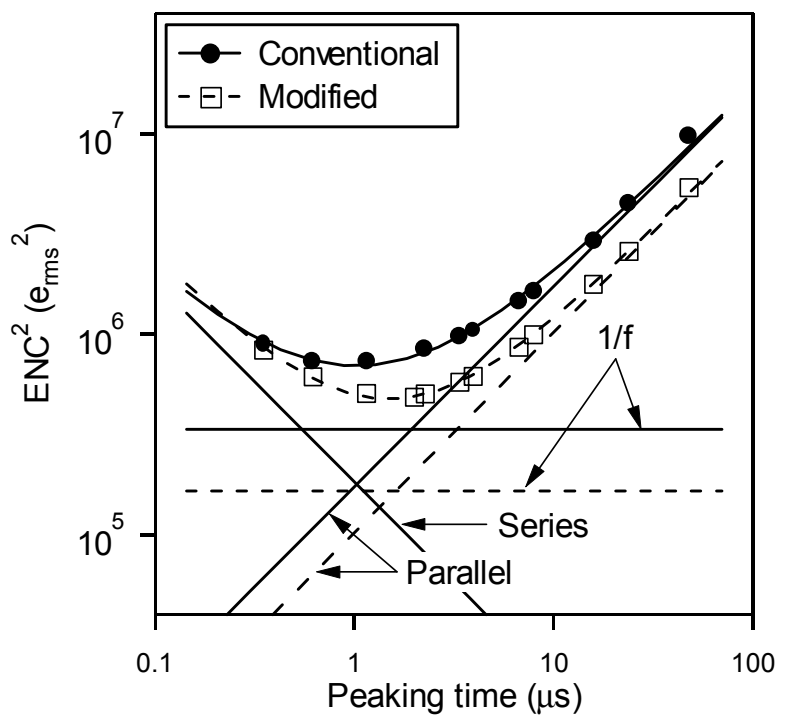

Fig. 3. Measured noise versus amplifier peaking time of a coplanar-grid detector fabricated using the conventional process and the modified process. The straight lines are the different noise components obtained by fitting to the data. with this detector before and after reprocessing the detector. The improvement in noise can be clearly seen from the pulser peak. The improvement in the $662 \mathrm{keV}$ gamma-ray peak is larger than expected if it is due to the reduction in noise alone. The difference is attributed to process variations that somehow affected the energy resolution. Improvement in gamma-ray resolution at low energy is also clearly seen in Fig. 5, with the $122 \mathrm{keV}$ and $136 \mathrm{keV}$ lines beginning to be resolved using the reprocessed detector. In this case, the

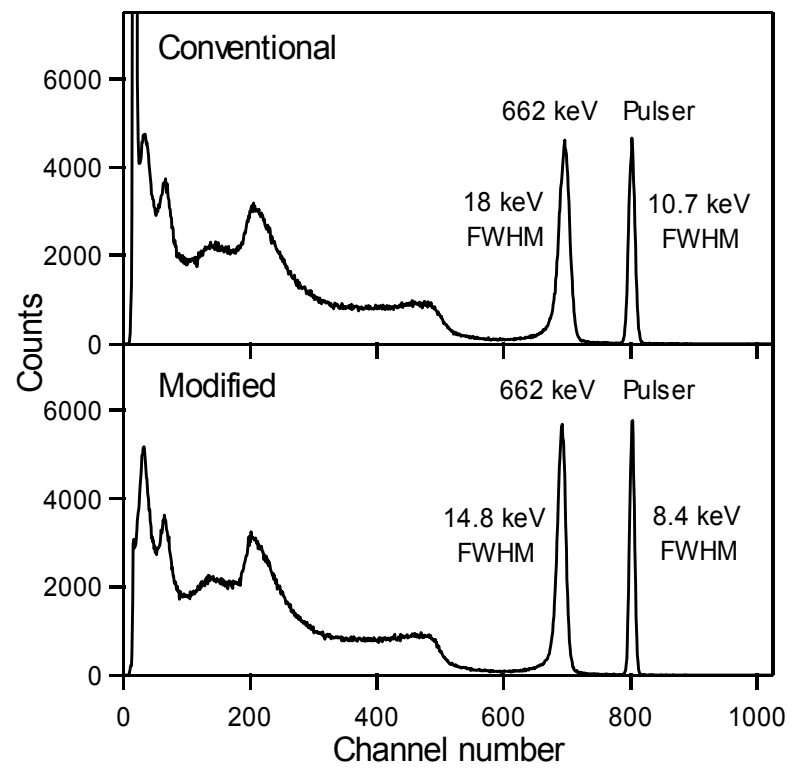

Fig. 4. Cs-137 spectra taken with the coplanar-grid detector fabricated using the conventional process (top) and the modified process (bottom). The amplifier peaking time was 4 $\mu \mathrm{s}$.

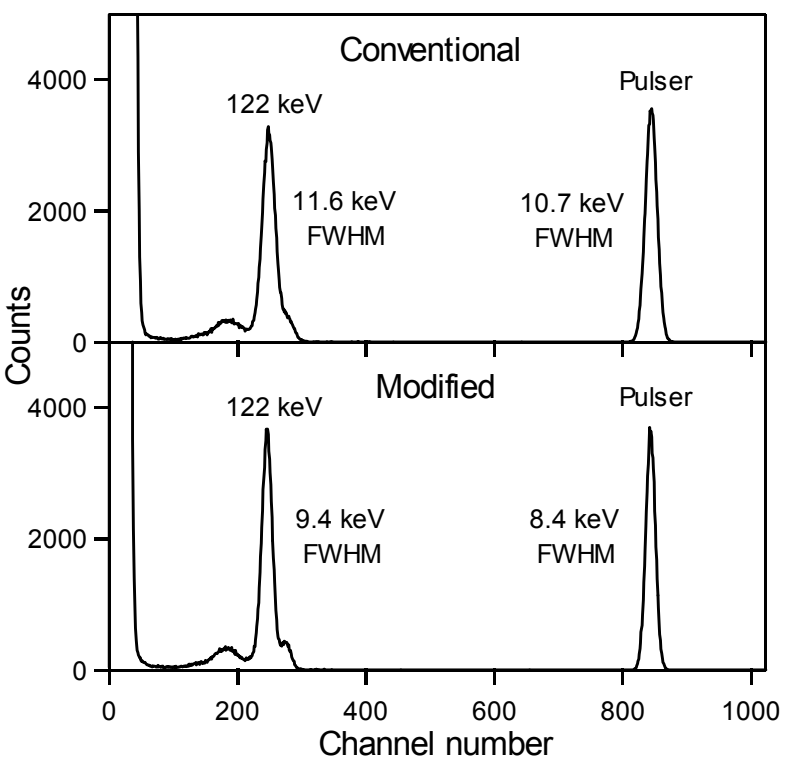

Fig. 5. Co-57 spectra taken with the coplanar-grid detector fabricated using the conventional process (top) and the modified process (bottom). The amplifier peaking time was 4 $\mu \mathrm{s}$. 
improvement is consistent with the reduction in noise. Similar improvement in noise has also been observed in a number of coplanar-grid detectors fabricated using this modified process.

\section{CONCLUSIONS}

The results from the coplanar-grid detectors show that the parallel noise associated with these detectors can be reduced significantly using a modified contact fabrication process. Based on our earlier measurements, this improvement is likely the result of a reduction in bulk current between the grid electrodes. A reduction in $1 / \mathrm{f}$ noise is also observed, which is attributed to the difference in treatments that the surface between the gird electrodes received as a result of the two different fabrication methods. We also showed that the evaporated gold contacts used on $\mathrm{CdZnTe}$ devices behave as Schottky barriers, which reduce bulk currents below that given by the bulk resistivity of the material. The bulk current is directly determined by the bulk resistivity only at sufficiently low bias voltages where the bulk current flow is not limited by the contact. Therefore, by using a guard ring device and observing the low-voltage I-V characteristic of the center contact, the bulk resistivity of the material can be properly determined.

\section{REFERENCES}

[1] P. N. Luke, M. Amman, J. S. Lee, B. A. Ludewigt, and H. Yaver, "A CdZnTe Coplanar-Grid Detector Array for Environmental Remediation," Nucl. Instr. Meth. A 458, 319 (2001).

[2] M. Amman, P. N. Luke, and J. S. Lee, "CdZnTe Material Uniformity and Coplanar-Grid Gamma-Ray Detector Performance," IEEE Trans. Nucl. Sci. 47, 760 (2000).

[3] P. N. Luke, M. Amman, J. S. Lee, and P. F. Manfredi, "Noise in CdZnTe Detectors," IEEE Trans. Nucl. Sci. 48(N3), 282 (2001).

[4] E. H. Rhoderick, Metal-Semiconductor Contacts, Clarendon Press, London, 1978. 